$\mathrm{T}$ he term "cor pulmonale" is still very popular in the medical literature, but its definition varies and there is presently no consensual definition. Forty years ago an expert committee of the World Health Organization' defined cor pulmonale as "hypertrophy of the right ventricle resulting from diseases affecting the function and/or structure of the lungs ...". This pathological definition is in fact of limited value in clinical practice. It has been proposed to replace the term "hypertrophy" by "alteration in the structure and function of the right ventricle". It has also been proposed to define clinically cor pulmonale by the presence of oedema in patients with respiratory failure. Finally, as pulmonary arterial hypertension is "the sine qua non" of cor pulmonale, ${ }^{2}$ we believe that the best definition of cor pulmonale is : pulmonary arterial hypertension resulting from diseases affecting the structure and/or the function of the lungs; pulmonary arterial hypertension results in right ventricular enlargement (hypertrophy and/or dilatation) and may lead with time to right heart failure.

A new diagnostic classification of pulmonary hypertension was developed by a group of experts in $1998^{3}$ and is presented on table 1 . In our opinion cor pulmonale corresponds to the third part of this classification (pulmonary hypertension associated with disorders of the respiratory system and/or hypoxaemia) and must be distinguished from pulmonary venous hypertension (part 2), and also from primary pulmonary hypertension (part 1 ) and from thromboembolic pulmonary hypertension (part 4).

This article reviews the current state of knowledge about pulmonary hypertension resulting from disorders of the respiratory system and/or from chronic hypoxaemia. We will only consider chronic cor pulmonale. Particular emphasis will be placed on chronic obstructive pulmonary disease (COPD) which is by far the main cause of cor pulmonale.

\title{
DEFINITIONS AND EPIDEMIOLOGY
}

Pulmonary hypertension complicating chronic respiratory disease is generally defined by the presence of a resting mean pulmonary artery pressure (PAP) $>20 \mathrm{~mm} \mathrm{Hg}$. This is slightly different from the definition of primary pulmonary hypertension (PAP $>25 \mathrm{mmHg}$ ). ${ }^{3}$ In young $(<50$ years) healthy subjects PAP is most often between $10-15 \mathrm{~mm} \mathrm{Hg}$. With aging there is a slight increase in PAP, by about $1 \mathrm{~mm} \mathrm{Hg} / 10$ years. A resting PAP $>20 \mathrm{~mm} \mathrm{Hg}$ is always abnormal. In the "natural history" of COPD, pulmonary hypertension is often preceded by an abnormally large increase in PAP during exercise, defined by a pressure $>30 \mathrm{~mm} \mathrm{Hg}$ for a mild level of steady state exercise. The term "exercising" pulmonary hypertension has been used by some authors, but the term "pulmonary hypertension" should be reserved for resting pulmonary hypertension.

Cor pulmonale is a common type of heart disease, as a result of its close association with COPD which has emerged, in recent years, as a leading cause of disability and death. ${ }^{4}$ But there are in fact very few data about the incidence and prevalence of cor pulmonale. The main reason is that right heart catheterisation cannot be performed on a large scale in patients at risk. An alternative approach is the use of non-invasive methods, particularly Doppler echocardiography. It should be possible to investigate large groups of respiratory patients with echo Doppler within the next few years.

A UK study performed in Sheffield ${ }^{5}$ has tried to determine the prevalence of patients at risk of developing pulmonary hypertension and cor pulmonale - that is, patients with hypoxaemic lung disease. In the study population, aged $\geqslant 45$ years, an estimated $0.3 \%$ had both an arterial oxygen tension $\left(\mathrm{PaO}_{2}\right)<7.3 \mathrm{kPa}(55 \mathrm{~mm} \mathrm{Hg})$ and a forced expiratory volume in one second $\left(\mathrm{FEV}_{1}\right)<50 \%$

Correspondence to: Professor Emmanue Weitzenblum, Service de Pneumologie, Hôpital de Hautepierre, 67098 Strasbourg Cedex, France; emmanuel.weitzenblum@ chru-strasbourg.fr of the predicted value. For England and Wales this could represent 60000 subjects at risk of pulmonary hypertension and eligible for long term oxygen therapy.

The mortality related to cor pulmonale is also difficult to assess. There are data about the mortality resulting from chronic lung disease ( 100 000/year in the USA ${ }^{4}$ ) but we do not know precisely the role of secondary pulmonary hypertension in this mortality. Pulmonary hypertension is a complication, among others, of advanced COPD and it is not possible to separate it from its causative diseases. 
Table 1 New diagnostic classification of pulmonary hypertension ${ }^{3}$

\section{Pulmonary arterial hypertension}

1.1 Primary pulmonary hypertension (a) Sporadic

(b) Familial

1.2 Related to

(a) Collagen vascular disease

(b) Congenital systemic to pulmonary shunts

(c) Portal hypertension

(d) HIV infection

(e) Drugs/toxins

(1) Anorexigens

(2) Other

(f) Persistent pulmonary hypertension of the newborn (g) Other

\section{Pulmonary venous hypertension}

2.1 Left sided atrial or ventricular heart disease

2.2 Left sided valvar heart disease

2.3 Extrinsic compression of central pulmonary veins

(a) Fibrosing mediastinitis

(b) Adenopathy/tumours

2.4 Pulmonary veno-occlusive disease

2.5 Other

3. Pulmonary hypertension associated with disorders of the respiratory system and/or hypoxaemia

3.1 Chronic obstructive pulmonary disease

3.2 Interstitial lung disease

3.3 Sleep disordered breathing

3.4 Alveolar hypoventilation disorders

3.5 Chronic exposure to high altitude

3.6 Neonatal lung disease

3.7 Alveolar capillary dysplasia

3.8 Other

4. Pulmonary hypertension caused by chronic thrombotic and/or embolic disease

4.1 Thromboembolic obstruction of proximal pulmonary arteries

4.2 Obstruction of distal pulmonary arteries

(a) Pulmonary embolism (thrombus, tumour, ova and/or

parasites, foreign material)

(b) In situ thrombosis

(c) Sickle cell disease

5. Pulmonary hypertension caused by disorders directly affecting the pulmonary vasculature

5. 1 Inflammatory

(a) Schistosomiasis

(b) Sarcoidosis

(c) Other

5.2 Pulmonary capillary haemangiomatosis

\section{AETIOLOGY: WHICH CHRONIC LUNG DISEASE MAY LEAD TO COR PULMONALE?}

Table 2 lists the chronic respiratory diseases which may lead to cor pulmonale. Primary pulmonary hypertension, pulmonary thromboembolic disease, and diseases of the pulmonary vascular bed have been excluded from this list which is far from exhaustive. There are three major groups of diseases:

- those characterised by a limitation to airflow (COPD and other causes of chronic bronchial obstruction)

- those characterised by a restriction of pulmonary volumes from extrinsic or parenchymatous origin (restrictive lung diseases)

- those where the relatively well preserved mechanical properties of the lungs and chest wall contrast with pronounced gas exchange abnormalities which are partially explained by poor ventilatory drive (respiratory insufficiency of "central" origin).

COPD is the major cause of chronic respiratory insufficiency and cor pulmonale, and it probably accounts for $80-90 \%$ of the
Table 2 Diseases of the respiratory system associated with pulmonary hypertension (except primary pulmonary hypertension, pulmonary thromboembolic disease, and diseases of the pulmonary vascular bed)

Obstructive lung diseases

- COPD* (chronic obstructive bronchitis, emphysema and their association)

- Asthma (with irreversible airway obstruction)

- Cystic fibrosist

Bronchiectasis

- Bronchiolitis obliterans

Restrictive lung diseases

- Neuromuscular diseases: amyotrophic lateral sclerosis, myopathy, bilateral diaphragmatic paralysis, etc

Kyphoscoliosist

- Thoracoplasty

- Sequelae of pulmonary tuberculosis

- Sarcoidosis

- Pneumoconiosis $\dagger$

- Drug related lung diseases

- Extrinsic allergic alveolitis

- Connective tissue diseases

- Idiopathic interstitial pulmonary fibrosist

- Interstitial pulmonary fibrosis of known origin

Respiratory insufficiency of "central" origin

- Central alveolar hypoventilation

- Obesity-hypoventilation syndrome† (formerly "Pickwickian syndrome")

- Sleep apnoea syndrome $†$

*Very frequent cause of pulmonary hypertension

†Relatively frequent cause of pulmonary hypertension.

cases. COPD includes chronic obstructive bronchitis and emphysema which are often associated. Among the restrictive lung diseases kyphoscoliosis, idiopathic pulmonary fibrosis, and pneumoconiosis are the main causes of cor pulmonale. Among the aetiologies of respiratory insufficiency of "central" origin the obesity-hypoventilation syndrome (formerly "Pickwickian syndrome") is a relatively frequent cause of cor pulmonale.

\section{MECHANISMS OF COR PULMONALE}

As stated above pulmonary hypertension is the "sine qua non" of cor pulmonale. Accordingly, the mechanisms of cor pulmonale are first those of pulmonary hypertension. In chronic respiratory diseases pulmonary hypertension results from increased pulmonary vascular resistance (PVR) whereas cardiac output and pulmonary "capillary" wedge pressure are normal; pulmonary hypertension is said to be precapillary.

The factors leading to an increased PVR in chronic respiratory disease are numerous but alveolar hypoxia is by far the most predominant, ${ }^{2}$ at least in COPD, kyphoscoliosis, and the obesity-hypoventilation syndrome. Two distinct mechanisms of action of alveolar hypoxia must be considered: acute hypoxia causes pulmonary vasoconstriction, and chronic longstanding hypoxia induces structural changes in the pulmonary vascular bed (pulmonary vascular remodelling).

Hypoxic pulmonary vasoconstriction (HPV) has been known since the studies in 1946 of Von Euler and Liljestrand on the cat. HPV explains the rise of PVR and PAP observed in humans, and in almost all species of mammals, during acute hypoxia. This vasoconstriction is localised in the small precapillary arteries. Its precise mechanism is not fully understood. The clinical situations which bear the closest analogy with acute hypoxic challenges are probably exacerbations of COPD 


\section{Abbreviations}

COPD: chronic obstructive pulmonary disease

$\mathrm{FEV}_{1}$ : forced expiratory volume in one second

HPV: hypoxic pulmonary vasoconstriction

LTOT: long term oxygen therapy

MRI: magnetic resonance imaging

$\mathrm{PaO}_{2}$ : arterial oxygen tension

$\mathrm{PaCO}_{2}$ : arterial carbon dioxide tension

PAP: pulmonary artery pressure

PVR: pulmonary vascular resistance

RHF: right heart failure

RVEF: right ventricular ejection fraction

leading to acute respiratory failure, and the sleep related episodes of worsening hypoxaemia.

Pulmonary hypertension is generally observed in respiratory patients exhibiting pronounced chronic hypoxaemia $\left(\mathrm{PaO}_{2}<55-60 \mathrm{~mm} \mathrm{Hg}\right)$. It is accepted that chronic alveolar hypoxia leads to remodelling of the pulmonary vascular bed (hypertrophy of the muscular media of the small pulmonary arteries, muscularisation of pulmonary arterioles, and intimal fibrosis) comparable to that observed in natives living at high altitude. This remodelling leads to elevation of PVR and to pulmonary hypertension. In fact the remodelling of the pulmonary vessels may be observed early in non-hypoxaemic COPD patients with mild disease severity.

Furthermore, other functional factors must be considered, namely hypercapnic acidosis and hyperviscosity caused by polycythaemia, but their role seems small when compared to that of alveolar hypoxia. In idiopathic pulmonary fibrosis the increase of PVR is caused by anatomical factors: loss of pulmonary vascular bed or compression of arterioles and capillaries by the fibrosing process.

Pulmonary hypertension increases the work of the right ventricle, which leads more or less rapidly to right ventricular enlargement (associating hypertrophy and dilatation) which can result in ventricular dysfunction (systolic, diastolic). Later, right heart failure (RHF) characterised by the presence of peripheral oedema can be observed, at least in some respiratory patients. The interval between the onset of pulmonary hypertension and the appearance of RHF is not known and may vary from one patient to another. There is a relation between the severity of pulmonary hypertension and the development of RHF.

\section{CLINICAL ASSESSMENT OF COR PULMONALE: PLACE OF NON-INVASIVE METHODS}

The clinical signs of cor pulmonale are relatively insensitive ${ }^{6}$ and some of them (signs related to an increased jugular venous pressure) are often obscured by hyperinflation of the chest $^{6}$ which is present in a number of COPD patients. Furthermore, the clinical signs occur late, being observed at an advanced stage of the disease far after the development of pulmonary hypertension. Peripheral (ankle) oedema is the best sign of RHF but it is not specific and can arise from other causes; in some patients with pulmonary hypertension, it does not occur at all. A murmur of tricuspid regurgitation, suggesting right ventricular dilatation, is a very late sign in respiratory patients. Accentuation of the pulmonary component of the second heart sound is only observed in patients with severe pulmonary hypertension.

The detection of right ventricular hypertrophy by electrocardiography has a high specificity but a very low sensitivity. A normal ECG does not exclude the presence of pulmonary hypertension, particularly in COPD patients. Similarly, the radiological signs of pulmonary hypertension (increased width of the right descending pulmonary artery) are poorly sensitive and the radiological appearance of a dilated right ventricle is a very late (and inconsistent) sign.

The non-invasive diagnosis of pulmonary hypertension is presently based on echocardiography. Continuous wave Doppler echocardiography allows the calculation of the transtricuspid pressure gradient from the peak velocity of the tricuspid regurgitant jet, by applying the Bernouilli equation. Assuming a right atrial pressure of $5 \mathrm{~mm} \mathrm{Hg}$, it is thus possible to calculate right ventricular systolic pressure (right atrial pressure + transtricuspid pressure gradient) which is identical to pulmonary artery systolic pressure. It is also possible to estimate the diastolic pulmonary artery pressure by summing the right atrial pressure and the end diastolic pressure gradient between the pulmonary artery and the right ventricle. Pulsed wave Doppler echocardiography, also based on the measurement of flow velocity, allows an indirect estimation of pulmonary artery systolic pressure. However, hyperinflation makes echocardiography difficult in many COPD patients and a reliable examination cannot be obtained in more than $60-80 \%$ of the cases. The good correlations that have been observed in cardiac patients between PAP estimated from echo data and pressures measured invasively have not always been confirmed in COPD patients ${ }^{7}$ and a mean error of the estimate, for PAP, of about $10 \mathrm{~mm} \mathrm{Hg}$ has been reported. ${ }^{7}$

Two dimensional echocardiography is used to measure right ventricular dimensions and the right ventricular wall thickness, making it possible to assess the presence of right ventricular hypertrophy and/or dilatation. However, magnetic resonance imaging (MRI) is probably the best method for measuring right ventricular dimensions because it produces the best images of the right ventricle. In COPD patients good correlations have been noted between right ventricular free wall volume measured by MRI and PAP. MRI is also a good method for detecting changes in right ventricular function, but it is expensive and available only in specialised centres.

Radionuclide ventriculography allows the measurement of right ventricular ejection fraction (RVEF). An RVEF < 40-45\% is considered abnormal, but RVEF is not a good index of right ventricular function; it gives only an estimate of the systolic function and is afterload dependent, decreasing when PAP and PVR increase. ${ }^{8}$ Accordingly, the decreased RVEF observed in many COPD patients is caused primarily by increased afterload conditions and is not an indicator of "true" right ventricular dysfunction.

\section{MAIN FEATURES OF PULMONARY HYPERTENSION IN CHRONIC RESPIRATORY DISEASE}

The main characteristic of pulmonary hypertension in chronic respiratory disease is probably its mild to moderate degree of hypertension, with resting PAP in a stable state of the disease ranging usually between $20-35 \mathrm{~mm} \mathrm{Hg}$. This modest degree of pulmonary hypertension, well recognised in COPD, is very different from left heart disease, congenital heart disease, pulmonary thromboembolic disease, and particularly primary pulmonary hypertension, where PAP is usually $>40-$ $50 \mathrm{~mm} \mathrm{Hg}$. Table 3 compares the pulmonary haemodynamic data of COPD patients ${ }^{9}$ with a large series of patients with primary pulmonary hypertension (US National Institutes of Health Registry). It can be seen that pulmonary hypertension is severe in primary pulmonary hypertension (mean (SD) PAP 
Table 3 Comparison of pulmonary hypertension in chronic hypoxic lung disease (COPD) to primary pulmonary hypertension

\begin{tabular}{lll} 
& PPH & COPD \\
\hline Number of patients & 187 & 62 \\
Number of women & 110 & 2 \\
Age (years) & $36(15)$ & $55(8)$ \\
$\mathrm{FEV}(\mathrm{ml})$ & & $1170(390)$ \\
$\mathrm{TLC}(\%$ of predicted) & & $110(15)$ \\
$\mathrm{PaO}_{2}(\mathrm{~mm} \mathrm{Hg})$ & $60(9)$ \\
$\mathrm{PaCO}_{2}(\mathrm{~mm} \mathrm{Hg})$ & & $45(6)$ \\
$\mathrm{PAP}(\mathrm{mm} \mathrm{Hg})$ & $60(15)$ & $26(6)$ \\
$\mathrm{PCP}(\mathrm{mm} \mathrm{Hg})$ & $8(4)$ & $8(2)$ \\
$\mathrm{Q}\left(\mathrm{l} / \mathrm{mm} / \mathrm{m}^{2}\right)$ & $2.27(0.90)$ & $3.8(1.1)$ \\
$\mathrm{PVR}\left(\mathrm{mm} \mathrm{Hg} / \mathrm{l} / \mathrm{min} / \mathrm{m}^{2}\right)$ & $26(14)$ & $4.8(1.4)$ \\
\hline
\end{tabular}

Values presented as mean (SD)

Primary pulmonary hypertension data are from the American $\mathrm{NIH}$

Registery; COPD data are from Weitzenblum et al. ${ }^{9}$

Registery; COPD data are from Weitzenblum et al. ${ }^{9}$
COPD, chronic obstructive pulmonary disease; $\mathrm{FEV}_{1}$, forced

expiratory volume in one second; PAP, pulmonary artery mean

pressure; $\mathrm{PCP}$, pulmonary capillary wedge pressure; $\mathrm{PPH}$, primary

pulmonary hypertension; $P V R$, pulmonary vascular resistance; $Q$,

cardiac output; TLC, total lung capacity.

60 (15) $\mathrm{mm} \mathrm{Hg}$ ) but is rather modest in COPD (PAP 26 (6) $\mathrm{mm} \mathrm{Hg}$ ). A PAP $\geqslant 40 \mathrm{~mm} \mathrm{Hg}$ is unusual in COPD patients except when they are investigated during an acute exacerbation or when there is an associated cardiopulmonary disease. The consequences of this modest level of pulmonary hypertension include the absence or late occurrence of RHF and the frequent inability of non-invasive methods to achieve a diagnosis of pulmonary hypertension. However, pulmonary hypertension, even if mild at baseline, may worsen during exercise and sleep and during acute exacerbations of the disease.

During steady state exercise PAP increases notably in advanced COPD patients with resting pulmonary hypertension, ${ }^{10}$ as illustrated in fig 1 which shows that in these patients (group 3) mean PAP rises from $27 \mathrm{~mm} \mathrm{Hg}$ to $55 \mathrm{~mm} \mathrm{Hg}$ during a 30-40 W exercise of 7-10 minutes duration. This is explained by the fact that PVR does not decrease during exercise in these patients; as the cardiac output is doubled for this level of exercise, PAP increases by about $100 \%$ (fig 1).

Acute increases of PAP during sleep have been observed in COPD patients with respiratory failure. ${ }^{11}$ They are principally observed in REM (rapid eye movement) sleep during which dips in oxygen saturation are more severe. These episodes of sleep related desaturation are not caused by apnoeas, except if COPD is associated with a sleep apnoea syndrome, but by alveolar hypoventilation and/or ventilation-perfusion mismatching. ${ }^{11}$ The more profound the dips of hypoxaemia, the more severe the peaks of pulmonary hypertension (PAP can increase by $>10 \mathrm{~mm} \mathrm{Hg}$ from its baseline value).

Episodes of acute respiratory failure are characterised by a worsening of hypoxaemia and hypercapnia, and simultaneously there is a pronounced increase in PAP. ${ }^{12}$ PAP may increase by as much as $20 \mathrm{~mm} \mathrm{Hg}$ but usually returns to its baseline after recovery, as shown in fig 2 . The striking parallel between changes in $\mathrm{PaO}_{2}$ and PAP suggests the presence of HPV. The acute increases of afterload occurring during exercise, sleep, and exacerbations of the disease can favour the development of RHF. ${ }^{13}$

On the other hand the progression of pulmonary hypertension is slow, at least in COPD patients, and PAP may remain stable over periods of $3-10$ years. ${ }^{14}$ In a series of 93 COPD

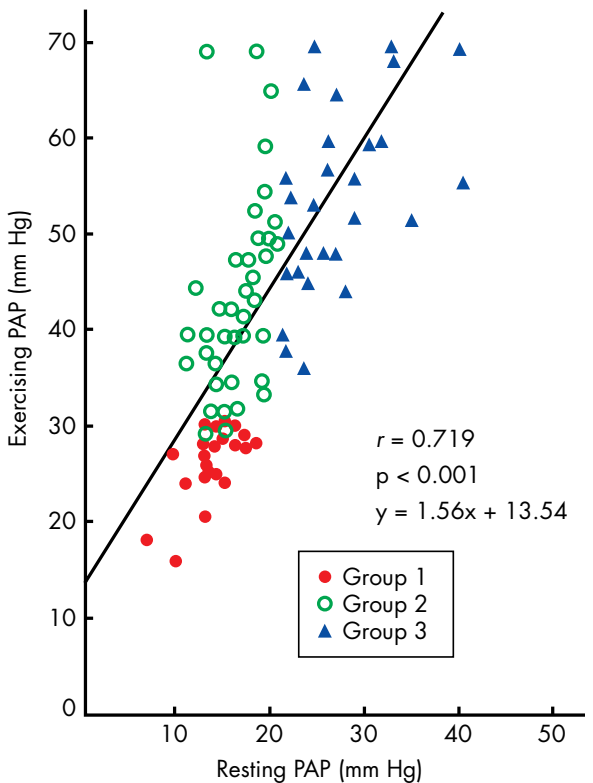

Figure 1 Resting and exercising pulmonary artery mean pressure (PAP) in a large series of chronic obstructive pulmonary disease patients (results are in $\mathrm{mm} \mathrm{Hg}$ ). Group 1: resting PAP $<20 \mathrm{~mm} \mathrm{Hg}$ and exercising PAP $<30 \mathrm{~mm} \mathrm{Hg}$. Group 2: resting PAP $<20 \mathrm{~mm}$ $\mathrm{Hg}$ and exercising PAP $>30 \mathrm{~mm} \mathrm{Hg}$. Group 3: resting PAP $>20$ $\mathrm{mm} \mathrm{Hg}$ (pulmonary hypertension).

patients followed up during a mean of 90 months, the average change in PAP was only $+0.5 \mathrm{~mm} \mathrm{Hg} / \mathrm{year}$ for the group as a whole. ${ }^{14}$ This means that in the majority of COPD patients whose PAP is initially normal $(<20 \mathrm{~mm} \mathrm{Hg}$ ) it will not exceed $20 \mathrm{~mm} \mathrm{Hg}$ after five years. But a minority (about 30\%) of advanced COPD patients exhibit a notable worsening of PAP during the follow up $^{14}$ : these patients do not differ from the others at the onset, but they are characterised by a progressive deterioration of $\mathrm{PaO}_{2}$ and $\mathrm{PaCO}_{2}$ during the evolution, which underlines the necessity for regular measurements of arterial blood gases for advanced COPD patients.

\section{FROM PULMONARY HYPERTENSION TO RIGHT HEART FAILURE}

The classical view of the development of RHF in chronic respiratory patients - that is, pulmonary hypertension leading to right ventricular enlargement, right ventricular dysfunction, and finally to RHF-has been questioned. ${ }^{6}$ That peripheral oedema, frequently observed in advanced COPD patients, reflects true RHF has been debated and even denied, in particular because the degree of pulmonary hypertension is often mild in COPD. ${ }^{15}$ Peripheral oedema is not synonymous with RHF and may simply indicate the presence of secondary hyperaldosteronism induced by functional renal insufficiency which is, in turn, a consequence of hypercapnic acidosis and/or hypoxaemia. ${ }^{615}$

The role of pressure overload in the development of RHF in these patients has also been debated since the observation that there was no difference in PAP between COPD patients with pronounced peripheral oedema and haemodynamic signs of RHF and other similar patients without oedema and without haemodynamic signs of RHF. ${ }^{16}$ However, some COPD patients with peripheral oedema do have RHF which is accounted for by a significant worsening of pulmonary hypertension during exacerbations of the disease (table 4 ). ${ }^{13}$ In nine of 16 COPD patients with pronounced peripheral oedema, 


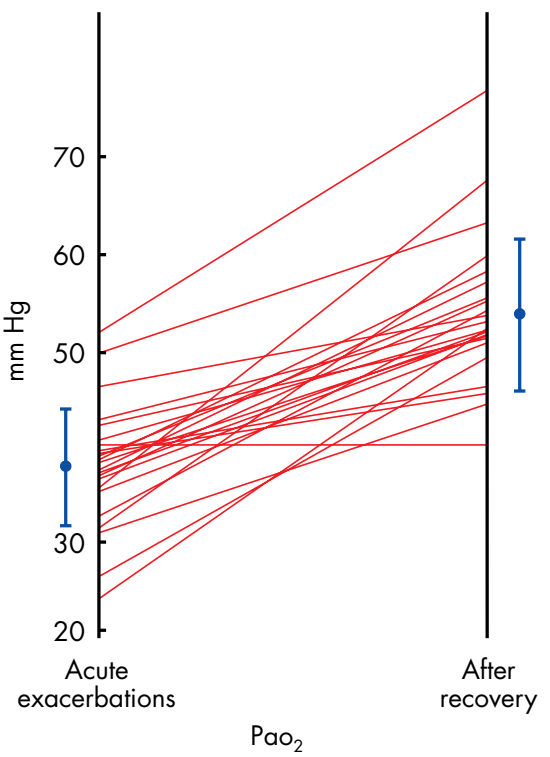

haemodynamic signs of RHF were present during an exacerbation of the disease with a worsening of hypoxaemia and hypercapnia. This worsening, as a result of raised HPV, probably explained the increase in PAP from baseline value and the development of RHF. ${ }^{13}$

Right ventricular contractility, assessed by the end systolic pressure-volume relation, is generally preserved in respiratory patients with pulmonary hypertension investigated when the disease is in a stable state. The only circumstances where a diminished right ventricular contractility could be documented in COPD patients are in acute exacerbations with presence of peripheral oedema and haemodynamic signs of RHF. $^{16}$

In summary, many patients with advanced COPD never develop RHF while, on the other hand, at least some patients experience episodes of true RHF during exacerbations of the disease accompanied by a worsening of pulmonary hypertension. Peripheral oedema is not synonymous with RHF and may simply indicate the presence of secondary hyperaldosteronism consequent to respiratory failure.

\section{PROGNOSIS OF COR PULMONALE}

The occurrence of documented RHF (peripheral oedema) was classically an indicator of poor prognosis in respiratory patients. In fact it is now accepted that a prolonged survival $(\geqslant 10$ years) can be observed after the first episode of peripheral oedema. The prevalence of clinical RHF has greatly decreased with the application of long term oxygen therapy (LTOT), with a resulting improvement in prognosis.
Figure 2 Evolution of arterial oxygen tension $\left(\mathrm{PaO}_{2}\right)$ and mean pulmonary artery pressure (PAP) in a series of chronic obstructive pulmonary disease patients investigated during acute exacerbations and after recovery. The pronounced improvement in $\mathrm{PaO}_{2}$ (from mean $38 \mathrm{~mm} \mathrm{Hg}$ to $53 \mathrm{~mm} \mathrm{Hg}$ ) is accompanied by a profound decrease in PAP (from mean $44 \mathrm{~mm}$ $\mathrm{Hg}$ to $27 \mathrm{~mm} \mathrm{Hg}$ ).
The level of PAP is a good indicator of prognosis in COPD ${ }^{917}$ but also in various categories of chronic respiratory disease such as idiopathic pulmonary fibrosis and sequelae of pulmonary tuberculosis. ${ }^{17}$ The prognosis is worse in COPD patients with pulmonary hypertension when compared to similar patients without pulmonary hypertension. ${ }^{9}$ In COPD patients with a mild degree of pulmonary hypertension (20$35 \mathrm{~mm} \mathrm{Hg}$ ) the five year survival rate is about $50 \% .^{9}{ }^{17}$ The prognosis is particularly poor for patients with severe pulmonary hypertension. ${ }^{17}$ LTOT greatly improves the survival of hypoxaemic COPD patients ${ }^{18} 19$ and, accordingly, the prognosis of pulmonary hypertension is improved by LTOT, which could partly be explained by the reduction of RHF episodes with LTOT. Of interest, PAP is still an excellent prognostic indicator in COPD patients receiving LTOT, probably because it is a good marker of both the duration and the severity of alveolar hypoxia in these patients.

\section{TREATMENT OF COR PULMONALE}

The treatment of RHF involves diuretics (most often frusemide (furosemide)) and oxygen therapy. Digitalis is used only in the case of an associated left heart failure or in the case of arrhythmia. The treatment of pulmonary hypertension includes vasodilators and LTOT. Is it really necessary to treat pulmonary hypertension in chronic hypoxic lung disease? Is the treatment of the disease itself (for example, COPD) not sufficient? Pulmonary hypertension is generally mild to moderate in COPD and the necessity for treating a mild hypertension can been questioned. An argument in favour of

Table 4 Evolution of arterial blood gases and haemodynamic variables before and during an episode of peripheral oedema in COPD patients

\begin{tabular}{|c|c|c|c|c|c|c|c|c|c|c|}
\hline & \multicolumn{2}{|c|}{ RVEDP $(\mathrm{mm} \mathrm{Hg})$} & \multicolumn{2}{|c|}{ PAP $(\mathrm{mm} \mathrm{Hg})$} & \multicolumn{2}{|c|}{$Q\left(1 / \mathrm{min} / \mathrm{m}^{2}\right)$} & \multicolumn{2}{|c|}{$\mathrm{PaO}_{2}(\mathrm{mmHg})$} & \multicolumn{2}{|c|}{$\mathrm{PaCO}_{2}(\mathrm{~mm} \mathrm{Hg})$} \\
\hline & $\mathrm{T1}$ & $\mathrm{T} 2$ & $\mathrm{~T} 1$ & $\mathrm{~T} 2$ & $\mathrm{~T} 1$ & $\mathrm{~T} 2$ & $\mathrm{~T} 1$ & $\mathrm{~T} 2$ & $\mathrm{~T} 1$ & T2 \\
\hline Group $1(n=9)$ & $7.5(3.9)$ & $13.4\left(1.2^{*}\right.$ & $27(5)$ & $40(6)^{*}$ & $3.23(0.82)$ & $3.19(1.07)$ & $63(4)$ & $49(7)^{*}$ & $46(7)$ & $59(14)^{*}$ \\
\hline Group $2(n=7)$ & $5.5(2.4)$ & $5.1(1.5)$ & $20(6)$ & $21(5)$ & $3.63(0.36)$ & $3.29(1.32)$ & $66(7)$ & $59(7)$ & $42(6)$ & $45(6)$ \\
\hline \multicolumn{11}{|c|}{$\begin{array}{l}\text { Values presented as mean (SD). } \\
\text { *Difference between T1 and T2 significant, } p<0.001 \text {. } \\
\text { Group 1, patients with haemodynamic signs of right heart failure (elevated RVEDP); Group 2, patients without haemodynamic signs of right heart failure. } \\
\text { PAP, pulmonary artery mean pressure. Q, cardiac output; RVEDP, right ventricular end diastolic pressure; T1, stable state of the disease; T2, episode of } \\
\text { oedema. Adapted from Weitzenblum et al. }{ }^{13}\end{array}$} \\
\hline
\end{tabular}




\section{Chronic cor pulmonale: key points}

- Cor pulmonale can be defined as pulmonary arterial hypertension resulting from diseases affecting the structure and/or function of the lungs. Pulmonary hypertension results in right ventricular enlargement and may lead with time to right heart failure

- Chronic obstructive pulmonary disease (COPD) is by far the main cause of chronic respiratory insufficiency and cor pulmonale

- In COPD alveolar hypoxia is the first cause of pulmonary hypertension. Acute hypoxia causes pulmonary vasoconstriction and chronic longstanding hypoxia induces pulmonary vascular remodelling

- In chronic respiratory disease pulmonary hypertension is of mild to moderate degree, but it may worsen during exercise, sleep, and acute exacerbations of the disease

- Many patients with advanced COPD will never develop right heart failure, but other patients experience episodes of right heart failure during exacerbations of the disease accompanied by a worsening of pulmonary hypertension

- Long term oxygen therapy is at present the best treatment for pulmonary hypertension in chronic respiratory failure. Future treatment may combine oxygen therapy and specific vasodilators

treatment is that pulmonary hypertension, even when modest during a stable period of the disease, may worsen, particularly during acute exacerbations, and these acute increases in PAP can contribute to the development of RHF. The best argument in favour of treatment is that LTOT, which is prescribed to very hypoxaemic respiratory patients, has favourable pulmonary haemodynamic effects.

Experience with vasodilator therapy has come from the treatment of primary and severe pulmonary hypertension. There are very few selective pulmonary vasodilators. At present, inhaled nitric oxide cannot be administered for long periods because of toxicological reasons. Prostacyclin, bosentan, and sildenafil, which are effective in treating patients with primary pulmonary hypertension, do not seem appropriate for COPD patients and there is, at present, no justification for the long term use of vasodilators in these patients.

One of the aims of LTOT in COPD patients is to attenuate the development of pulmonary hypertension and to reduce the frequency of episodes of RHF. Since alveolar hypoxia is the major determinant of the rise of PVR and PAP, it is logical to treat with LTOT hypoxaemic COPD patients exhibiting pulmonary hypertension. The NOTT (nocturnal oxygen therapy trial) ${ }^{18}$ and Medical Research Council ${ }^{19}$ multicentre studies have shown that LTOT improves ${ }^{18}$ or at least stabilises $^{19}$ pulmonary hypertension. LTOT has also been shown to reverse the progression of pulmonary hypertension in COPD patients. ${ }^{20}$ However, PAP rarely returns to normal. The longer the period of LTOT ( $>16$ hours/day) the better the haemodynamic results. At present LTOT is the best treatment for pulmonary hypertension in COPD patients. In the future, this treatment may combine LTOT and specific vasodilators.

\section{REFERENCES}

1 Anon. Chronic cor pulmonale. Report of an expert committee. Circulation 1963;27:594-15

2 Fishman AP. Chronic cor pulmonale. Am Rev Respir Dis

$1978 ; 114: 775-94$.
- A state of the art report on chronic cor pulmonale by one of the major experts in this field.

3 Rich S, ed. Primary pulmonary hypertension: executive summary from the World Symposium - Primary Pulmonary Hypertension 1998. Available from the World Health Organization. URL: http://www.who. int/ncd/cvd/pph.html

- Report of an expert panel on "primary pulmonary hypertension", with presentation of the new diagnostic classification of pulmonary hypertension.

4 Pauwels RA, Buist AS, Calverley PMA, et al, on behalf of the GOLD Scientific Committee. Global strategy for the diagnosis, management, and prevention of chronic obstructive pulmonary disease. NHLBI/WHO global initiative for chronic obstructive lung disease (GOLD) workshop summary. Am J Respir Crit Care Med 2001;163:1256-76.

- Report of a very recent consensus workshop organised by National Heart, Lung, and Blood Institute and the World Health Organization on the definition, classification of severity, burden, and management of COPD.

5 Williams BT, Nicholl JP. Prevalence of hypoxaemic chronic obstructive lung disease with reference to long-term oxygen therapy. Lancet $1985 ; i: 369-72$.

6 MacNee W. Pathophysiology of cor pulmonale in chronic obstructive pulmonary disease. Am J Respir Crit Care Med 1994;150:833-52; $1158-68$.

- A very comprehensive review of the pathophysiology of pulmonary hypertension and right heart failure in COPD.

7 Tramarin R, Torbicki A, Marchandise B, et al. Doppler echocardiographic evaluation of pulmonary artery pressure in chronic obstructive pulmonary disease. A European multicentre study. Eur Heart J 1991;12:103-11.

8 Brent BN, Berger HJ, Matthay RA, et al. Physiologic correlates of right ventricular ejection fraction in chronic obstructive pulmonary disease: a combined radionuclide and hemodynamic study. Am J Cardiol 1982;50:255-62.

9 Weitzenblum E, Hirth C, Ducolone A, et al. Prognostic value of pulmonary artery pressure in chronic obstructive pulmonary disease. Thorax 1981;36:752-8.

- This article shows that pulmonary arterial hypertension is a major prognostic factor in patients with advanced COPD.

10 Horsfield R, Segel N, Bishop JM. Pulmonary circulation in chronic bronchitis at rest and during exercise breathing air or $80 \%$ oxygen. Clin Sci 1968:43:473-83.

11 Fletcher EC, Levin DC. Cardiopulmonary hemodynamics during sleep in subjects with chronic obstructive pulmonary disease: the effect of short and long-term oxygen. Chest 1984;85:6-14.

- This article shows that episodes of sleep related hypoxaemia, particularly during REM sleep, induce peaks of pulmonary hypertension in COPD patients.

12 Abraham AS, Cole RB, Green ID, et al. Factors contributing to the reversible pulmonary hypertension of patients with acute respiratory failure studied by serial observations during recovery. Circ Res 1969;24:51-60.

- This article describes and explains the reversibility of pulmonary arterial hypertension during recovery following acute exacerbations in COPD patients.

13 Weitzenblum E, Apprill M, Oswald M, et al. Pulmonary hemodynamics in patients with chronic obstructive pulmonary disease before and during an episode of peripheral edema. Chest 1994;105:1377-82.

14 Weitzenblum E, Sautegeau A, Ehrhart M, et al. Long-term course of pulmonary arterial pressure in chronic obstructive pulmonary disease. Am Rev Respir Dis 1984;130:993-8.

- This article shows that the long term progression of pulmonary hypertension is slow in most COPD patients and is linked to the evolution of hypoxaemia.

15 Richens JM, Howard P. Oedema in cor pulmonale. Clin Sci 1982:62:255-9

16 MacNee W, Wathen C, Flenley DC, et al. The effects of controlled oxygen therapy on ventricular function in patients with stable and decompensated cor pulmonale. Am Rev Respir Dis 1988;137:1289-95.

17 Bishop JM, Cross KW. Physiological variables and mortality in patients with various categories of chronic respiratory disease. Bull Eur Physiopathol Respir 1984;20:495-500.

18 Nocturnal Oxygen Therapy Trial Group. Continuous or nocturnal oxygen therapy in hypoxemic chronic obstructive lung disease. Ann Intern Med 1980;93:391-8.

- This landmark study has shown that continuous oxygen therapy, compared to nocturnal oxygen therapy, given to very hypoxaemic COPD patients, improves both survival and pulmonary hypertension.

19 Medical Research Council Working Party. Long-term domiciliary oxygen therapy in chronic hypoxic cor pulmonale complicating chronic bronchitis and emphysema. Lancet 1981;i:681-6.

- Another landmark study showing that long term domiciliary oxygen therapy, given for 15 hours/day in very hypoxaemic COPD patients, improves survival in comparison to conventional treatment.

20 Weitzenblum E, Sautegeau A, Ehrhart $M$, et al. Long term oxygen therapy can reverse the progression of pulmonary hypertension in patients with chronic obstructive pulmonary disease. Am Rev Respir Dis 1985;131:493-8. 